\title{
Productivity and Growth of Short-internode Muskmelon Plants at Various Spacings or Densities
}

\author{
Dean E. Knavel \\ Department of Horticulture and Landscape Architecture, University of Kentucky Lexington, \\ KY 40546
}

\begin{abstract}
Additional index words. Cucumis melo, fruit yield, total leaf area, shaded leaf area, dry weight, cantaloupe
Abstract. Short-internode (SI) muskmelon (Cucumis melo L.) genotypes Ky-P $\mathbf{P}_{7}$ (si-1 gene for SI) and Main Dwarf (si-3 gene for SI) were compared with the normal-internode (NI) cultivar Mainstream at various plant spacings or planting densities over 3 years. SI 'Honey Bush' (si-1 gene for SI) and 'Bush Star' (si-1 gene for SI) were included in 2 years. At double the population, SI plants ( $s i$ gene type) produced $\approx 35 \%$ fewer fruit than 'Mainstream' plants grown at one-half the population density. Spacing generally had no effect on average fruit weight, but increasing plant density of SI genotypes decreased the number of fruit per plant. Generally, doubling the density reduced leaf area and total plant dry weight, but had minimal effect on the amount of shaded leaf area. Ky- ${ }_{7}$, 'Honey Bush', and 'Bush Star' plants had more leaf shading than 'Mainstream' and Main Dwarf plants.
\end{abstract}

Muskmelon yields might be increased through the development of SI or bush cultivars grown at higher planting densities than commonly used for NI-length cultivars (Davis et al., 1976; Kalb and Davis, 1984; Mohr and Knavel, 1966). Several SI (si1 gene) cultivars are commercially available, but their yielding potential has not been explored. Knavel (1988) previously reported that SI (si-1 gene) $\mathrm{Ky}-\mathrm{P}_{7}$ plants grown at double the plant population of NI 'Mainstream' plants produced only one-half the number and total weight of fruit. Since fruit size was similar for both plant types when grown at the same spacing, it was concluded that the lower yields for $\mathrm{Ky}-\mathrm{P}_{7}$ plants were mainly due to fewer fruiting sites and less leaf area per plant exposed to solar radiation than for 'Mainstream' plants. Nutritional and irrigation studies (unpublished data) indicated that lower yields by SI plants were not associated with reduced nutrient and/or water uptake.

The objective of this study was to further evaluate the-effect of spacing or planting densities on growth and yield of SI genotypes and to compare these variables with the response of the NI 'Mainstream'.

\section{Materials and Methods}

Fruit yield and plant growth data were recorded in 1987 through 1989 for SI genotypes Ky- $\mathrm{P}_{7}$ and Main Dwarf and the NI 'Mainstream'. SI cultivars Honey Bush and Bush Star were included in 1988 and 1989, respectively. In this study, the SI gene si-3 controls shortened internodes in Main Dwarf, while the si-1 gene controls them in Ky- $\mathrm{P}_{7}$, 'Honey Bush', and 'Bush Star'.

Each year, plants were started in the greenhouse and grown in a peat-perlite medium in $2.5 \times 3.8 \times 3.8-\mathrm{cm}$ plastic cell pots until transplanted $\approx 4$ weeks later onto 1.2 -m-wide, black polyethylene mulch-covered beds in the field. Nitrogen was applied from ammonium nitrate at the rate of $112 \mathrm{~kg} \cdot \mathrm{ha}^{-1}$ and rototilled into the soil before the mulch was laid. Phosphorus and $\mathrm{K}$ were applied similarly from superphosphate and potassium sulfate, respectively, when needed according to soil tests

Received for publication 10 Dec. 1990. This Kentucky Agricultural Experiment Station paper (90-10-168) is published by permission of the Director. The cost of publishing this paper was defrayed in part by the payment of page charges. Under postal regulations, this paper therefore must be hereby marked advertisement solely to indicate this fact. and Kentucky Experimental Station recommendations. Weekly sprays for pest control followed recommendations for growing muskmelons in Kentucky (Roberts et al., 1988). The soil type at the Univ. of Kentucky farm was a Maury silt-loam (fine, mixed, mesic, Typic Paleudalfs).

Spacing treatments for SI plants in 1987 and 1988 were single rows of one per hill $0.6 \mathrm{~m}$ apart (12 plants/replicate), two per hill $0.6 \mathrm{~m}$ apart (24 plants/replicate), one per hill $0.3 \mathrm{~m}$ apart (24 plants/replicate), and plants offset in double rows of one per hill $0.6 \mathrm{~m}$ apart, with double rows $0.3 \mathrm{~m}$ apart (24 plants/ replicate). The spacing for 'Mainstream' plants in 1987 and 1988 was one per hill $0.6 \mathrm{~m}$ apart in a single row (12 plants/ replicate). In 1989, spacing treatments for all genotypes were single rows of one per hill, either 0.6 or $0.3 \mathrm{~m}$ apart. The plastic mulch plots were spaced $1.8 \mathrm{~m}$ apart, center to center. Treatments were randomized in complete blocks in 1987 and 1988 and in a split-plot design in 1989, with three replications each year. Plots were not irrigated.

Leaf area was recorded with a LI-COR (Model 3100; LICOR, Lincoln, Neb.) meter on 72-day-old plants in 1987 and 1989, and 86-day-old plants in 1988. The amount of leaf shading was determined by spraying diluted, white, latex paint over four plants of each genotype at each spacing in each replication. After the paint was dry, the two inner sprayed plants were taken for growth data, leaving eight plants per treatment for yield data. Leaves were cut from the vine at the junction of the petiole and lamina and separated into painted and unpainted. For leaves showing partially painted areas, painted portions were cut from the unpainted portions. The percentage of shading was based on the area of leaves without paint to total leaf area. For dry weight, leaves, stem, and petioles were oven-dried at 70C. Before the vines were dried in 1987, two plants per replication of each genotype grown at the $0.6-\mathrm{m}$ spacing were measured for growth comparison. Data were tested by analysis of variance.

For soil moisture data, soil samples were collected from the center of the row by taking six probes to a depth of $15 \mathrm{~cm}$ through the plastic mulch midway between plants within each replication of each treatment. Moisture was determined gravimetrically. Data collected for percentages of soil moisture and leaf shading were analyzed following arcsin transformation.

Abbreviations: NI, normal internode; SI, short internode. 
Table 1. Effect of plant spacing on number and weight of muskmelon fruit in 1987.

\begin{tabular}{|c|c|c|c|c|}
\hline \multirow[b]{4}{*}{ Genotype } & \multicolumn{4}{|c|}{ Planting arrangement ${ }^{z}$} \\
\hline & \multicolumn{3}{|c|}{ Single row } & \multirow{2}{*}{$\begin{array}{c}\text { Double row, } \\
\text { staggered }\end{array}$} \\
\hline & \multirow{2}{*}{$\begin{array}{c}0.6 \mathrm{~m} \\
\text { (one/hill) }\end{array}$} & \multirow{2}{*}{$\begin{array}{c}0.3 \mathrm{~m} \\
\text { (one/hill) }\end{array}$} & \multirow{2}{*}{$\begin{array}{c}0.6 \mathrm{~m} \\
\text { (two/hill) }\end{array}$} & \\
\hline & & & & $0.6 \times 0.3 \mathrm{~m}$ \\
\hline \multirow{4}{*}{$\begin{array}{l}\text { Mainstream } \\
\text { Main Dwarf } \\
\mathrm{Ky}_{-} \mathrm{P}_{7}\end{array}$} & \multicolumn{4}{|c|}{ No. fruit/planty } \\
\hline & $6.6 \mathrm{a}$ & $\mathrm{NA}^{\mathrm{x}}$ & NA & NA \\
\hline & $6.4 \quad \mathrm{a}$ & $4.8 \mathrm{~b}$ & $4.3 \mathrm{~b}$ & $4.6 \mathrm{~b}$ \\
\hline & $3.0 \mathrm{c}$ & $2.0 \mathrm{~d}$ & $2.0 \mathrm{~d}$ & $2.2 \mathrm{~d}$ \\
\hline $\begin{array}{l}\text { Mainstream } \\
\text { Main Dwarf } \\
\mathrm{Ky}-\mathrm{P}_{7}\end{array}$ & $\begin{array}{l}1.05 \mathrm{a} \\
0.61 \mathrm{~b} \\
1.00 \mathrm{a}\end{array}$ & $\begin{array}{l}\text { Avg } w \\
\text { NA } \\
0.53 \mathrm{~b} \\
0.93 \mathrm{a}\end{array}$ & $\begin{array}{l}(\mathrm{kg} / \text { fruit }) \\
\mathrm{NA} \\
0.54 \mathrm{~b} \\
0.93 \mathrm{a}\end{array}$ & $\begin{array}{l}\text { NA } \\
0.50 \mathrm{~b} \\
0.95 \mathrm{a}\end{array}$ \\
\hline $\begin{array}{l}\text { Mainstream } \\
\text { Main Dwarf } \\
{\mathrm{Ky}-\mathrm{P}_{7}}\end{array}$ & $\begin{array}{ll}58.9 & \mathrm{~b} \\
58.1 & \mathrm{~b} \\
26.9 & \mathrm{c} \\
\end{array}$ & $\begin{array}{l}\text { No. fru } \\
\text { NA } \\
87.7 \text { a } \\
36.3 \text { c } \\
\end{array}$ & $\begin{array}{l}(1000 / h a) \\
\text { NA } \\
78.7 \text { a } \\
36.3 \mathrm{c} \\
\end{array}$ & $\begin{array}{l}\text { NA } \\
83.5 \text { a } \\
40.5 \text { c } \\
\end{array}$ \\
\hline \multicolumn{5}{|c|}{$\begin{array}{l}{ }^{\mathrm{z}} \text { Mean separation within rows and columns by Dunca } \\
\text { test, } P=0.05 \text {. } \\
{ }^{\mathrm{y}} \text { Means for seven harvests from } 22 \text { July to } 17 \text { Aug. } \\
{ }^{\mathrm{x}} \mathrm{NA}=\text { treatment not applied. }\end{array}$} \\
\hline \multicolumn{5}{|c|}{$\begin{array}{l}\text { Table } 2 . \text { Effect of plant spacing on number and weight of muskmelon } \\
\text { fruit in } 1988 .\end{array}$} \\
\hline \multirow[b]{4}{*}{ Genotype } & \multicolumn{4}{|c|}{ Planting arrangement ${ }^{z}$} \\
\hline & \multicolumn{3}{|c|}{ Single row } & Double row, \\
\hline & \multirow{2}{*}{$\begin{array}{c}0.6 \mathrm{~m} \\
\text { (one/hill) }\end{array}$} & \multirow{2}{*}{$\begin{array}{c}0.3 \mathrm{~m} \\
\text { (one/hill) }\end{array}$} & \multirow{2}{*}{$\begin{array}{c}0.6 \mathrm{~m} \\
\text { (two/hill) }\end{array}$} & staggered \\
\hline & & & & $0.6 \times 0.3 \mathrm{~m}$ \\
\hline & \multicolumn{4}{|c|}{ No. fruit/planty } \\
\hline Mainstream & $10.5 \mathrm{a}$ & $\mathrm{NA}^{\mathbf{x}}$ & NA & NA \\
\hline Main Dwarf & $9.1 \mathrm{a}$ & $6.2 \mathrm{~b}$ & $6.1 \mathrm{~b}$ & $6.1 \mathrm{~b}$ \\
\hline $\mathrm{Ky}-\mathrm{P}_{7}$ & $4.5 \mathrm{c}$ & $3.9 \mathrm{~cd}$ & 2.9 ef & $4.0 \mathrm{~cd}$ \\
\hline \multirow[t]{2}{*}{ Honey Bush } & $3.6 \mathrm{de}$ & $4.0 \mathrm{~cd}$ & $2.6 \mathrm{f}$ & $3.1 \mathrm{ef}$ \\
\hline & \multicolumn{4}{|c|}{ Avg wt (kg/fruit) } \\
\hline Mainstream & $1.07 \mathrm{a}$ & NA & NA & NA \\
\hline Main Dwarf & $0.59 \mathrm{c}$ & $0.53 \mathrm{c}$ & $0.52 \mathrm{c}$ & $0.47 \mathrm{c}$ \\
\hline $\mathrm{Ky}-\mathrm{P}_{7}$ & $0.83 \mathrm{~b}$ & $0.78 \mathrm{~b}$ & $0.74 \mathrm{~b}$ & $0.77 \mathrm{~b}$ \\
\hline \multirow[t]{2}{*}{ Honey Bush } & 0.99 a & $0.79 \mathrm{~b}$ & $0.80 \mathrm{~b}$ & $0.79 \mathrm{~b}$ \\
\hline & \multicolumn{4}{|c|}{ No. fruit $(1000 / h a)$} \\
\hline Mainstream & $92.2 \mathrm{~b}$ & $\mathrm{NA}$ & NA & NA \\
\hline Main Dwarf & $81.6 \mathrm{c}$ & $112.2 \mathrm{a}$ & $109.8 \mathrm{a}$ & $109.8 \mathrm{a}$ \\
\hline $\mathrm{Ky}-\mathrm{P}_{7}$ & $40.8 \mathrm{fg}$ & $69.6 \mathrm{~d}$ & $51.6 \mathrm{~d}$ & $72.0 \mathrm{~cd}$ \\
\hline Honey Bush & $32.4 \mathrm{~g}$ & $71.4 \mathrm{~cd}$ & $46.8 \mathrm{df}$ & $56.4 \mathrm{~d}$ \\
\hline
\end{tabular}

${ }^{\mathrm{z}}$ Mean separation within rows and columns by Duncan's multiple range test, $P=0.05$.

${ }^{\mathrm{y}}$ Means for 12 harvests from 16 July to 16 Sept.

${ }^{\mathrm{x}} \mathrm{NA}=$ treatment not applied.

\section{Results}

NI 'Mainstream' produced more fruit per plant and per unit growing area than SI si-1 genotype (Ky- $\mathrm{P}_{7}$, 'Honey Bush', and 'Bush Star') plants, regardless of spacing or planting density (Tables 1-3). At double the population, SI si-3 (Main Dwarf) plants produced a similar number of fruit per plant but more fruit per area than 'Mainstream' plants.

Plant spacing had a minimal effect on average fruit weight. In 2 of 3 years, average weights of 'Mainstream' and $\mathrm{Ky}-\mathrm{P}_{7}$ fruit were similar (Tables 1 and 3), and planting density had no effect on average weight of $\mathrm{Ky}-\mathrm{P}_{7}$ (Tables 2 and 3) and 'Bush Star' (Table 3) fruit. In 1988 (Table 2), average fruit weights of 'Mainstream' and 'Honey Bush' were similar but higher than

Table 3. Effect of plant spacing on number and weight of muskmelon fruit in 1989.

\begin{tabular}{lcc}
\hline \hline & \multicolumn{3}{c}{ Plant spacing } \\
\cline { 2 - 3 } Genotype & $\begin{array}{c}0.6 \mathrm{~m} \\
\text { (one/hill) }\end{array}$ & $\begin{array}{c}0.3 \mathrm{~m} \\
\text { (one/hill) }\end{array}$ \\
\hline Mainstream & \multicolumn{3}{c}{ No. fruit/plant $^{2}$} \\
Main Dwarf & 5.8 & 3.6 \\
Ky-P & 5.0 & 3.8 \\
Bush Star & 1.8 & 1.4 \\
Significance & 1.4 & 1.5 \\
$\quad$ Spacing & & $* *$ \\
$\quad$ Genotype & \multicolumn{3}{c}{$* *$} \\
$\quad$ Spacing $\times$ genotype & \multicolumn{3}{c}{$*$}
\end{tabular}

\section{Mainstream \\ Main Dwarf \\ $\mathrm{Ky}-\mathrm{P}_{7}$ \\ Bush Star \\ Significance \\ Spacing \\ Genotype \\ Spacing $\times$ genotype}

Mainstream

Main Dwarf

$\mathrm{Ky}-\mathrm{P}_{7}$

Bush Star

Significance

Spacing

Genotype

Spacing $\times$ genotype

${ }^{\mathrm{z}}$ Means for five harvests from 10 Aug. to 1 Sept.

$\mathrm{Ns}_{*}, * *$ Nonsignificant or significant $\mathrm{F}$ value at $P=0.05$ and 0.001 , respectively.

Table 4. Effect of plant spacing on leaf area and percentage of leaf area shaded for muskmelon plants 72 days after seeding in 1987.

\begin{tabular}{|c|c|c|c|c|}
\hline \multirow[b]{4}{*}{ Genotype } & \multicolumn{4}{|c|}{ Planting arrangement ${ }^{\mathrm{z}}$} \\
\hline & \multicolumn{3}{|c|}{ Single row } & \multirow{2}{*}{$\begin{array}{c}\text { Double row } \\
\text { staggered }\end{array}$} \\
\hline & \multirow{2}{*}{$\begin{array}{c}0.6 \mathrm{~m} \\
\text { (one/hill) }\end{array}$} & \multirow{2}{*}{$\begin{array}{c}0.3 \mathrm{~m} \\
\text { (one/hill) }\end{array}$} & \multirow{2}{*}{$\begin{array}{c}0.6 \mathrm{~m} \\
\text { (two/hill) }\end{array}$} & \\
\hline & & & & $0.6 \times 0.3 \mathrm{~m}$ \\
\hline & \multicolumn{4}{|c|}{ Leaf area $\left(1000 \mathrm{~cm}^{2} /\right.$ plant $)$} \\
\hline Mainstream & $19.2 \mathrm{a}$ & $\mathrm{NA}^{\mathrm{y}}$ & NA & NA \\
\hline Main Dwarf & $8.4 \mathrm{c}$ & $5.6 \mathrm{~d}$ & $5.6 \mathrm{~d}$ & $4.4 \mathrm{e}$ \\
\hline $\mathrm{Ky}-\mathrm{P}_{7}$ & $10.5 \mathrm{~b}$ & $11.3 \mathrm{~b}$ & $7.5 \mathrm{c}$ & $7.9 \mathrm{c}$ \\
\hline & \multicolumn{4}{|c|}{ Leaf area shaded $(\%)^{\mathrm{x}}$} \\
\hline Mainstream & 28 & NA & NA & NA \\
\hline Main Dwarf & 26 & $35 \mathrm{~cd}$ & $37 \mathrm{c}$ & $31 \mathrm{de}$ \\
\hline $\mathrm{Ky}-\mathrm{P}_{7}$ & $49 \mathrm{~b}$ & $53 \mathrm{ab}$ & $52 \mathrm{ab}$ & $55 \mathrm{a}$ \\
\hline
\end{tabular}

${ }^{\mathrm{z}}$ Mean separation within rows and columns by Duncan's multiple range test, $P=0.05$.

${ }^{\mathrm{y}} \mathrm{NA}=$ treatment not applied.

${ }^{\mathrm{x}}$ Actual data (transformed to arcsin before analysis).

for $\mathrm{Ky}-\mathrm{P}_{7}$ fruit from plants grown in single rows at one per hill spaced $0.6 \mathrm{~m}$ apart; doubling the plant population reduced the average weight of 'Honey Bush' fruit (Table 2). Main Dwarf fruit weighed considerably less than fruit of 'Mainstream' and other SI genotypes and was not affected by spacing.

Generally, doubling the plant populations reduced total leaf area and total dry weight of SI plants. Ky- $\mathrm{P}_{7}$ plants had $45 \%$ less leaf area than 'Mainstream' in 1987 (Table 4), but total leaf 
areas of 'Mainstream' and $\mathrm{Ky}-\mathrm{P}_{7}$ plants growing $0.6 \mathrm{~m}$ apart in single rows were similar in 1988 (Table 5) and 1989 (Table 6). Plants of all SI genotypes grown at double the plant population averaged $38 \%$ less leaf area than single plants grown $0.6 \mathrm{~m}$ apart (Tables 4-6). In 1989, 'Mainstream' plants grown at double the plant population had $32 \%$ less leaf area than plants grown $0.6 \mathrm{~m}$ apart (Table 6).

The average size of matured leaves from 105-day-old-plants grown at the lowest plant population in 1988 was 132, 138, 102, 90, and $53 \mathrm{~cm}^{2}$ for Ky- $\mathrm{P}_{7}$, 'Honey Bush', 'Bush Star', 'Mainstream', and Main Dwarf, respectively. This sequence indicates a distinct difference in leaf size and canopy architecture among genotypes. These sizes were smaller than for matured leaves from 70-day-old plants grown at the same spacing in 1989, which were 200, 96, and $60 \mathrm{~cm}^{2}$ for $\mathrm{Ky}-\mathrm{P}_{7}$, 'Mainstream', and Main Dwarf, respectively. This difference is at-

Table 5. Effect of plant spacing on leaf area, percentage of leaf area shaded, and total plant dry weight for muskmelon plants 86 days after seeding in 1988.

\begin{tabular}{|c|c|c|c|c|c|}
\hline \multirow[b]{4}{*}{ Genotype } & \multicolumn{5}{|c|}{ Planting arrangement ${ }^{z}$} \\
\hline & \multicolumn{4}{|c|}{ Single row } & \multirow{2}{*}{$\begin{array}{c}\text { Double row, } \\
\text { staggered }\end{array}$} \\
\hline & \multirow{2}{*}{\multicolumn{2}{|c|}{$\begin{array}{c}0.6 \mathrm{~m} \\
\text { (one/hill) }\end{array}$}} & \multirow{2}{*}{$\begin{array}{c}0.3 \mathrm{~m} \\
\text { (one/hill) }\end{array}$} & \multirow{2}{*}{$\begin{array}{c}0.6 \mathrm{~m} \\
\text { (two/hill) }\end{array}$} & \\
\hline & & & & & $0.6 \times 0.3 \mathrm{~m}$ \\
\hline & \multicolumn{5}{|c|}{ Leaf area $\left(1000 \mathrm{~cm}^{2} /\right.$ plant $)$} \\
\hline Mainstream & \multicolumn{2}{|c|}{$12.7 \mathrm{a}$} & $\mathrm{NA}^{\mathrm{y}}$ & NA & NA \\
\hline Main Dwarf & \multicolumn{2}{|c|}{$6.1 \mathrm{e}$} & $4.7 \mathrm{f}$ & $5.0 \mathrm{f}$ & $3.9 \mathrm{~g}$ \\
\hline $\mathrm{Ky}-\mathrm{P}_{7}$ & \multicolumn{2}{|c|}{$13.2 \mathrm{a}$} & $8.8 \mathrm{c}$ & $5.0 \mathrm{f}$ & $8.2 \mathrm{~cd}$ \\
\hline \multirow[t]{2}{*}{ Honey Bush } & \multicolumn{2}{|c|}{$10.1 \mathrm{~b}$} & $7.8 \mathrm{~d}$ & $6.3 \mathrm{e}$ & $6.3 \mathrm{e}$ \\
\hline & \multicolumn{5}{|c|}{ Leaf area shaded $(\%)^{\mathbf{x}}$} \\
\hline Mainstream & & $\mathrm{f}$ & NA & NA & NA \\
\hline Main Dwarf & & $\mathrm{e}$ & 25 ef & 33 & 39 \\
\hline $\mathrm{Ky}-\mathrm{P}_{7}$ & & $\mathrm{a}$ & 42 bc & 47 & 42 bc \\
\hline \multirow[t]{2}{*}{ Honey Bush } & 43 & $\mathrm{~b}$ & $35 \mathrm{~d}$ & 41 bc & $32 \mathrm{~d}$ \\
\hline & \multicolumn{5}{|c|}{ Total dry wt (g/plant) } \\
\hline Mainstream & 143 & $\mathrm{a}$ & NA & NA & NA \\
\hline Main Dwarf & 79 & $\mathrm{~d}$ & $64 \mathrm{e}$ & $60 \mathrm{e}$ & $74 \mathrm{~d}$ \\
\hline $\mathrm{Ky}-\mathrm{P}_{7}$ & 118 & $\mathrm{~b}$ & $95 \mathrm{c}$ & $57 \mathrm{e}$ & $74 \mathrm{~d}$ \\
\hline Honey Bush & 106 & c & $101 \mathrm{c}$ & $76 \mathrm{~d}$ & $57 \mathrm{e}$ \\
\hline
\end{tabular}

${ }^{\mathrm{z}}$ Mean separation within rows and columns by Duncan's multiple range test, $P=0.05$.

${ }^{\mathrm{y}} \mathrm{NA}=$ treatment not applied.

${ }^{\mathrm{z}}$ Actual data (transformed to arcsin before analysis).

Table 6. Effect of plant spacing on leaf area, percentage of leaves shaded, and total plant dry weight for muskmelon plants 72 days after seeding in 1989. Both spacings, one plant per hill.

\begin{tabular}{|c|c|c|c|c|c|c|}
\hline \multirow[b]{2}{*}{ Genotype } & \multicolumn{2}{|c|}{$\begin{array}{c}\text { Total leaf area } \\
\left(1000 \mathrm{~cm}^{2} /\right. \\
\text { plant })\end{array}$} & \multicolumn{2}{|c|}{$\begin{array}{c}\text { Leaf area } \\
\text { shaded } \\
(\%)^{z}\end{array}$} & \multicolumn{2}{|c|}{$\begin{array}{c}\text { Total dry wt } \\
\text { (g/plant) }\end{array}$} \\
\hline & $0.6 \mathrm{~m}$ & $0.3 \mathrm{~m}$ & $0.6 \mathrm{~m}$ & $0.3 \mathrm{~m}$ & $0.6 \mathrm{~m}$ & $0.3 \mathrm{~m}$ \\
\hline Mainstream & 15.5 & 10.5 & 37 & 34 & 187 & 126 \\
\hline Main Dwarf & 10.8 & 7.0 & 38 & 42 & 91 & 75 \\
\hline $\mathrm{Ky}-\mathrm{P}_{7}$ & 17.0 & 11.1 & 63 & 56 & 136 & 91 \\
\hline Bush Star & 11.8 & 5.4 & 51 & 43 & 140 & 93 \\
\hline \multicolumn{7}{|l|}{ Significance } \\
\hline Spacing & \multicolumn{2}{|c|}{$* *$} & \multicolumn{2}{|c|}{ NS } & \multicolumn{2}{|c|}{$* *$} \\
\hline Genotype & \multicolumn{2}{|c|}{$* *$} & \multicolumn{2}{|c|}{$* *$} & \multicolumn{2}{|c|}{$* *$} \\
\hline Spacing $\times$ genotype & \multicolumn{2}{|c|}{ NS } & \multicolumn{2}{|c|}{ NS } & \multicolumn{2}{|c|}{ NS } \\
\hline
\end{tabular}

${ }^{\mathrm{z}}$ Actual data (transformed to arcsin before analysis).

$\mathrm{NS}, *, * *$ Nonsignificant or significant $\mathrm{F}$ value at $P=0.05$ and 0.01 , respectively. tributed to improved environmental conditions and faster growth in 1989 as compared to the hot dry conditions in 1988.

Plants with the si- 1 gene for SI had a higher percentage of their total leaf area shaded by younger leaves than did 'Mainstream' plants, and increasing plant population had only a slight effect on leaf shading. Leaf shading for Main Dwarf plants was similar to that for 'Mainstream' and considerably less than for other SI plants grown $0.6 \mathrm{~m}$ apart at one per hill. However, doubling the plant population of Main Dwarf plants, by planting two per hill $0.6 \mathrm{~m}$ apart or one per hill $0.3 \mathrm{~m}$ apart in 1987 (Table 2) or two per hill or staggered in double rows in 1988 (Table 4), resulted in more of the leaf area being shaded than for plants grown at $0.6 \mathrm{~m}$ apart one per hill.

At all sampling times, plots with SI plants of si-1 genotypes had the same or higher soil moisture than plots with 'Mainstream' plants, regardless of spacing (Table 7). At other sampling dates, 19 June and 29 July 1987 and 28 July 1988, soil moisture was similar for all plots, with an average of $18 \%, 13 \%$, $6 \%$, and $21 \%$, respectively.

\section{Discussion}

The lower fruit yield of SI Ky- $\mathrm{P}_{7}$, 'Honey Bush', and 'Bush Star' plants in comparison with NI 'Mainstream' plants is partially attributable to reduced vine growth, as indicated by a lower dry weight, less total leaf area, and greater leaf shading. The lower fruit-setting capacity of $\mathrm{Si} \mathrm{Ky}-\mathrm{P}_{7}$ plants is attributed to smaller vines and less secondary branching than for SI Main Dwarf and 'Mainstream' plants (Fig. 1). The number of nodes (leaves) and immature fruit on SI Ky- $\mathrm{P}_{7}$, plants were about onehalf the number for NI 'Mainstream' plants of the same age (Knavel, 1988). Nerson et al. (1983) reported that plants of SI cultivars produced fewer perfect flowers than plants of the NI cultivar.

Table 7. Influence of plant spacing on soil moisture (\%) in 1987, 1988 , and 1989

\begin{tabular}{|c|c|c|c|c|}
\hline \multirow[b]{4}{*}{ Genotype } & \multicolumn{4}{|c|}{ Planting arrangement ${ }^{z, y}$} \\
\hline & \multicolumn{3}{|c|}{ Single row } & \multirow{2}{*}{$\begin{array}{c}\text { Double row, } \\
\text { staggered }\end{array}$} \\
\hline & \multirow{2}{*}{$\begin{array}{c}0.6 \mathrm{~m} \\
\text { (one/hill) }\end{array}$} & \multirow{2}{*}{$\begin{array}{c}0.3 \mathrm{~m} \\
\text { (one/hill) }\end{array}$} & \multirow{2}{*}{$\begin{array}{c}0.6 \mathrm{~m} \\
\text { (two/hill) }\end{array}$} & \\
\hline & & & & $0.6 \times 0.3 \mathrm{~m}$ \\
\hline & \multicolumn{4}{|c|}{30 June 1987} \\
\hline Mainstream & $17.3 \mathrm{de}$ & $N_{A}{ }^{\mathbf{x}}$ & NA & NA \\
\hline Main Dwarf & $18.3 \mathrm{c}$ & $19.1 \mathrm{~b}$ & $16.8 \mathrm{e}$ & $17.4 \mathrm{~d}$ \\
\hline \multirow[t]{2}{*}{$\mathrm{Ky}-\mathrm{P}_{7}$} & $19.4 \mathrm{~b}$ & $20.2 \mathrm{a}$ & $19.7 \mathrm{ab}$ & $19.5 \mathrm{~b}$ \\
\hline & \multicolumn{4}{|c|}{8 July 1988} \\
\hline Mainstream & $10.5 \mathrm{bc}$ & NA & NA & NA \\
\hline Main Dwarf & $11.4 \mathrm{a}$ & $11.2 \mathrm{ab}$ & $11.1 \mathrm{ab}$ & $10.3 \mathrm{c}$ \\
\hline $\mathrm{Ky}-\mathrm{P}_{7}$ & $11.6 \mathrm{a}$ & $10.7 \mathrm{bc}$ & $10.0 \mathrm{c}$ & $10.2 \mathrm{c}$ \\
\hline Honey Bush & $11.9 \mathrm{a}$ & $10.6 \mathrm{bc}$ & $11.0 \mathrm{ab}$ & $10.7 \mathrm{bc}$ \\
\hline & \multicolumn{4}{|c|}{11 Aug. 1989} \\
\hline Mainstream & 18.1 & 20.9 & NA & NA \\
\hline Main Dwarf & 18.2 & 20.3 & NA & NA \\
\hline $\mathrm{Ky}-\mathrm{P}_{7}$ & 23.3 & 24.6 & NA & NA \\
\hline Bush Star & 23.6 & 22.6 & NA & NA \\
\hline \multicolumn{5}{|l|}{ Significance } \\
\hline Genotype & \multicolumn{3}{|c|}{$* *$} & \\
\hline Spacing & \multirow{2}{*}{\multicolumn{3}{|c|}{$\begin{array}{l}* \\
* *\end{array}$}} & \\
\hline Genotye $\times$ spacing & & & & \\
\hline
\end{tabular}



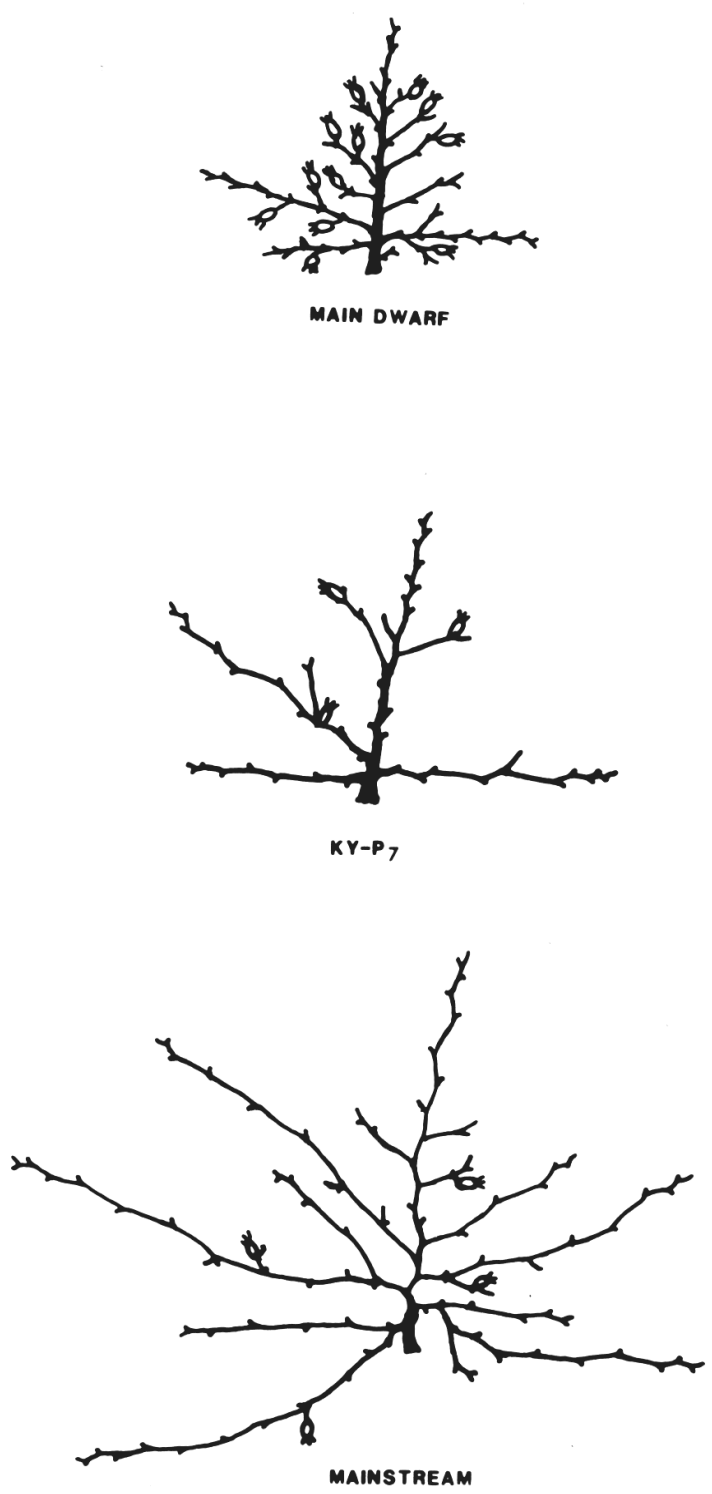

Fig. 1. Vine architecture for 72-day-old Main Dwarf (top), Ky- $\mathrm{P}_{7}$, (center), and 'Mainstream' (bottom) plants grown in 1987.
Even though $\mathrm{Ky}-\mathrm{P}_{7}$, had the same leaf area as 'Mainstream' in 1988 and 1989 for plants in single rows $0.6 \mathrm{~m}$ apart, nearly one-half of the total leaf area was shaded. Due to the SI plant's architecture (Fig. 1), the large newly matured leaves of si-1 genotypes are close together, overlap, and shade the older, inner leaves. In contrast, SI Main Dwarf and NI 'Mainstream' plants have more stem branching and smaller leaves, a combination that allows more of its total leaf area to be exposed to sunlight for potentially greater photosynthetic activity and, subsequently, greater potential for supporting female flower production and fruit set. Acock et al. (1990) found that older leaves of NI muskmelon plants had low net assimilation rates, and fruit growth depended on a certain number of leaves and their potential for carrying on photosynthesis.

Cultivars with the si-1 gene for SI used in these studies, regardless of spacing, produced significantly fewer fruit and lower total fruit weights than did 'Mainstream' plants. Main Dwarf plants set as many fruit as 'Mainstream' plants, but of lesser size. However, these characteristics might be improved in Main Dwarf-type plants through breeding. In conclusion, I recommend that growers continue to use commercial NI instead of SI cultivars at spacings appropriate for their area.

\section{Literature Cited}

Acock, B., M.C. Acock, and D. Pasternak. 1990. Interactions of $\mathrm{CO}_{2}$ enrichment and temperature on carbohydrate production and accumulation in muskmelon leaves. J. Amer. Soc. Hort Sci. 11.5:525529.

Davis, D.W., M.A. Shehata, and C.J. Eide. 1976. Minnesota 266 muskmelon breeding line. HortScience 11:273.

Kalb, T.J. and D.W. Davis. 1984. Evaluation of combining ability, heterosis and genetic variance for yield, maturity, and plant characteristics in bush muskmelon. J. Amer. Soc. Hort. Sci. 109:416419.

Knavel, D.E. 1988. Growth, development, and yield potential of shortinternode muskmelon. J. Amer. Soc. Hort. Sci. 113:595-599.

Mohr, H.C. and D.E. Knavel. 1966. Progress in the development of short-internode (bush) cantaloupes. HortScience 1:16.

Nerson, H., H.S. Paris, and Z. Karchi. 1983. Characteristics of birdsnest-type muskmelons. Scientia Hort. 21:341-352.

Roberts, C.R., J. Strang, W. Dunwell, T. Jones, L. Townsend, J. Hartman, D. Hershman, J.R. Martin, J.D. Green, and W. Thorn. 1988. Commercial vegetable crop recommendations-1988-89. Univ. of Kentucky Ser. Publ. Id-36. 